\title{
Essais
}

Revue interdisciplinaire d'Humanités

\section{Le mémorial du Bomber Command à Londres}

Quand les Britanniques retrouvent la mémoire

\section{Madeline Zielinski}

\section{(2) OpenEdition}

Journals

Édition électronique

URL : http://journals.openedition.org/essais/5731

DOI : 10.4000/essais.5731

ISSN : 2276-0970

Éditeur

École doctorale Montaigne Humanités

\section{Édition imprimée}

Date de publication : 15 janvier 2015

Pagination : 94-109

ISBN : 978-2-9544269-4-5

ISSN : 2417-4211

Référence électronique

Madeline Zielinski, «Le mémorial du Bomber Command à Londres », Essais [En ligne], 6 | 2015, mis en ligne le 30 novembre 2020, consulté le 04 décembre 2020. URL : http://journals.openedition.org/ essais/5731 ; DOI : https://doi.org/10.4000/essais.5731 


\section{Le mémorial du Bomber Command à Londres. Quand les Britanniques retrouvent la mémoire}

\section{Madeline Zielinski}

Le mémorial en l'honneur des anciens combattants du Bomber Command, la force de bombardement de la Royal Air Force, fut inauguré en grande pompe le 28 juin 2012 à Londres par la reine Elizabeth II en personne, sous le regard de plusieurs milliers d'invités ayant eu le privilège d'être conviés à cet événement de grande ampleur. Ce mémorial est la première commémoration officielle en Grande-Bretagne du sacrifice des équipages du Bomber Command ayant pris part à la Seconde Guerre mondiale. Cette reconnaissance tardive peut au premier abord sembler surprenante ; en effet, la Grande-Bretagne a longtemps tenté d'oublier - ou tout du moins d'évoquer le moins possible - le souvenir des bombardements massifs auxquels le Bomber Command a participé, principalement en Allemagne, à partir de 1942. Cette page de l'histoire de la guerre, et du questionnement moral qui en découle, entache le souvenir glorieux d'épisodes héroïques tels que la Bataille d'Angleterre - pour Churchill, "l'heure de gloire " ("Britain's finest hour ") du pays - au cours de laquelle les chasseurs du Fighter Command, le corps d'aviation de chasse britannique, repoussèrent vaillamment les attaques de la Luftwaffe, forçant Hitler à mettre un terme à ses plans d'invasion de la Grande-Bretagne. Aujourd'hui encore, la fierté des Britanniques d'avoir résisté seuls pendant près d'un an alors que les démocraties en Europe s'écroulaient les unes après les autres, demeure palpable et vivace. Le souvenir des bombardements en Allemagne est, quant à lui, bien plus problématique dans la mémoire collective britannique. Les attaques répétées du Bomber Command entrainèrent de nombreuses victimes parmi les populations civiles ; près de 600000 Allemands auraient, d'après les estimations des historiens, perdu la vie dans les bombardements ${ }^{1}$. Ceux-ci visaient principalement les villes industrielles allemandes de la Ruhr dans le but d'anéantir le potentiel de guerre ennemi. Par ailleurs, le débat fait toujours rage chez les historiens

1 John A. Gentry, How Wars are Won and Lost. Vulnerability and Military Power, Santa Barbara, ABC CLIO, 2012, p. 63. 
quant à la nature des cibles visées par les bombardiers ; certains estiment qu’à plusieurs reprises, la valeur stratégique des bombardements était discutable, et que ceux-ci visaient délibérément des zones civiles. C'est le cas par exemple du bombardement de Dresde les 13, 14 et 15 février 1945, qui suscite aujourd'hui encore beaucoup d'émotion et des débats houleux en Grande-Bretagne et en Allemagne. À la suite de la controverse de Dresde en 1945, les équipages du Bomber Command et leurs chefs furent critiqués et tombèrent en disgrâce en raison du rôle qu'ils avaient joué dans une campagne militaire dont l'utilité et la moralité étaient sans cesse remises en question. La question de la moralité des bombardements demeure l'une des grandes controverses de la Seconde Guerre mondiale en raison de la stratégie adoptée par les bombardiers britanniques et américains, qui consistait à faire de villes entières et leurs habitants les cibles de leurs attaques massives aux bombes incendiaires. Dans un tel contexte, la commémoration des anciens combattants du Bomber Command peut sembler pour le moins inconfortable ; pourtant, en 2008, une campagne de grande ampleur, obtenant le soutien du plus grand nombre, fut lancée en GrandeBretagne pour l'édification d'un mémorial en leur honneur. Longtemps oubliés, ignorés ou marginalisés, les anciens du Bomber Command sont désormais élevés au rang de héros. Ce revirement de situation ne peut que susciter l'interrogation quant aux facteurs ou événements ayant engendré ce changement. L'analyse qui suit tentera d'y apporter des réponses.

Lorsque la guerre fut déclarée en 1939, le Bomber Command ne disposait que de 272 appareils, dont certains n'étaient pas opérationnels. La flotte aérienne était composée de bombardiers bimoteurs à moyen rayon d'action aux performances décevantes ${ }^{2}$. Au mois de juin 1938, Neville Chamberlain, alors Premier ministre britannique, pour qui le gouvernement se devait d'adopter une position claire sur la conduite d'un conflit aérien, déclarait à la Chambre des Communes que le recours aux bombardements dans le but de démoraliser les populations civiles était exclu. Il précisa :

C'est absolument contraire à la loi internationale, et j'ajouterais que, selon moi, c'est une politique erronée du point de vue de ceux qui l'adoptent, mais je ne crois pas que des attaques délibérées sur une population civile permettront jamais à ceux qui font la guerre de la gagner ${ }^{3}$.

Churchill lui-même, influencé par Anthony Éden, était initialement opposé aux bombardements de villes entières ; il l'avait d'ailleurs exprimé en 1932, lorsqu'il avait recommandé que le gouvernement britannique propose

2 Chris Chant, Allied Bombers 1939-1945, Minneapolis, Amber Books, 2008, p. 28.

3 Steven Garrett, Ethics and Airpower in World War IIII: The British Bombing of German Cities, New-York, St Martin's Press, 1997, p. 29. "This is absolutely contrary to international law, and I would add that, in my opinion, it is a mistaken policy from the point of view of those who adopt it, but I do not believe that the deliberate attacks upon a civilian population will ever win a war for those who make them. » 
des règles, lors de la conférence sur le Désarmement de Genève afin de limiter les bombardements aériens à des cibles militaires. Il semble que Churchill ait épousé la logique de dissuasion qui avait dominé les débats des théoriciens militaires dans les années 1930. Sa position changea lorsqu'il devint Premier ministre et que, selon l'historien militaire Basil Liddle-Hart, l'une des premières décisions de son gouvernement fut d'étendre les bombardements aux zones non-combattantes ${ }^{4}$.

La grande difficulté rencontrée par la Grande-Bretagne en 1939 était qu’elle n'avait pas les moyens d'avoir recours au bombardement de précision sur l'Allemagne - les équipages n'étaient pas correctement formés, et la RAF n'avait pas d'avion de bombardement à longue portée ni de bombe suffisamment lourde. Les résultats du Bomber Command demeuraient décevants ; ils remirent sérieusement en question la stratégie du bombardement de précision initialement décidée par la RAF et de sa faisabilité. Les objectifs du Bomber Command glissèrent peu à peu vers le bombardement de zones urbaines, et sur les dommages collatéraux que cela pourrait causer au moral des populations civiles 5 .

Le 14 février 1942, la Directive № 22 fut envoyée au Bomber Command. L'offensive de bombardement devait se concentrer sur le moral de la population civile ennemie et en particulier sur les travailleurs dans les usines. Au cas où le moindre doute subsisterait au quartier général du Bomber Command, le chef d'état-major envoya cette précision le jour suivant :

En référence à la nouvelle directive sur les bombardements : j'imagine qu'il est clair que les cibles visées seront des zones construites, et non pas, par exemple, les chantiers navals ou les usines d'avions... Cela doit être clarifié si ce n'est pas déjà compris ${ }^{6}$.

La décision d'adopter le bombardement massif - autrement dit, la dévastation systématique des villes allemandes - après le mois de février 1942 constitue une évolution dans la conduite de la guerre, et plus particulièrement dans le concept de la guerre totale. De 1942 à 1945, les trois quarts des bombardements sur l'Allemagne furent des bombardements massifs et non de précision.

Le recours aux bombardements fut pendant longtemps le seul moyen pour la Grande-Bretagne d'atteindre l'Allemagne et son industrie de guerre. Après 1942, avec la nomination du maréchal de l'Air Arthur Harris au commande-

4 John DiJoseph, Noble Cause Corruption, the Banality of Evil, and the Threat to American Democracy 1950-2008, Lanham, University Press of America, 2010, p. 4.

5 Michael Burleigh, Moral Combat. A History of World War Two, Londres, Harper Press, 2011, p. 487-488.

6 Steven Garrett, "The Bombing Campaign: the RAF ", in Primoratz Igor, Terror from the Sky. The Bombing of German Cities in World War II, Oxford, Berghahn Books, 2010, p. 27. " Ref the new bombing directive: I suppose it is clear that the aiming points are to be the built-up areas, not, for instance, the dockyards or aircraft factories... This must be made quite clear if it is not already understood. " 
ment du Bomber Command et le développement d'appareils de bombardement lourd plus performants, les attaques sur l'Allemagne s'intensifièrent. Harris était un personnage déterminé, parfois abrupt, trait de caractère qui lui attira les foudres de certains membres du gouvernement. Il était cependant très apprécié des membres d'équipage du Bomber Command, qui lui sont toujours restés fidèles et l'ont toujours farouchement défendu, et ce même lorsque, après la fin de la guerre, il est devenu la cible des critiques les plus violentes. Son apparente froideur et sa détermination à mettre l'Allemagne à genoux en la bombardant lui avaient valu, pendant la guerre, son surnom de "Butch », diminutif de «Butcher », le boucher.

Le 21 septembre 1943, Churchill déclarait à la Chambre des communes : "pour [détruire le régime nazi] nous ne reculerons devant aucune forme de violence $»^{7}$. Pourtant, le gouvernement craignait l'opinion publique, c'est pourquoi la décision de bombarder les civils allemands fut dissimulée aux Britanniques jusqu'en avril 1944, lorsque J.-M. Spaight, premier secrétaire du ministère de l'Air, fit la déclaration suivante :

Parce que nous n'avions aucune certitude sur les effets psychologiques de la déformation par la propagande du fait que c'était bien nous qui avions commencé l'offensive stratégique de bombardements, nous avons évité de donner à la décision majeure du 11 mai 1940 la visibilité qu'elle méritait. Cela était assurément une erreur. C'était une grande décision. Elle était aussi héroïque, aussi pleine de sacrifice de soi que la décision de la Russie d'adopter la politique de la "terre brûlée ". Cela a permis à Coventry et Birmingham, Sheffield et Southampton, de regarder Kiev et Kharkov, Stalingrad et Sébastopol dans les yeux. Nos alliés soviétiques auraient été moins critiques de notre inactivité de 1942 s'ils comprenaient ce que nous avions accompli ${ }^{8}$.

Harris, quant à lui, n'était pas favorable à ce que le gouvernement dissimule à la population les objectifs réels du Bomber Command. Contrairement au ministère de l'Air, Harris était convaincu que l'opinion publique britannique accepterait la stratégie du Bomber Command. Et en effet, l'opinion publique britannique était généralement favorable aux opérations de Bomber Command, elles-mêmes présentées favorablement dans les bulletins d'informations de la BBC. Le programme de la BBC qui rencontra le plus de succès pendant la guerre fut celui consacré au Bomber Command. Le programme suivait le journaliste Wynford Vaughan Thomas, qui accompagna le 4 septembre 1943 un

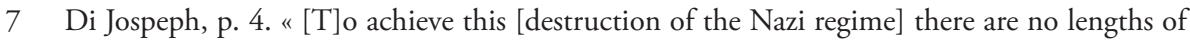
violence to which we will not go. »

8 Ibid. "Because we were doubtful about the psychological effect of propagandistic distortion of the truth that it was we who started the strategic bomber offensive, we have shrunk from giving our great decision of May 11, 1940, the publicity which it deserved. That, surely was a mistake. It was a splendid decision. It was as heroic, as self-sacrificing as Russia’s decision to adopt her policy of "scorched earth". It gave Coventry and Birmingham, Sheffield and Southampton, the right to look Kiev and Kharkov, Stalingrad and Sebastopol in the face. Our Soviet allies would have been less critical of our inactivity of 1942 if they understood what we had done. " 
escadron de Lancasters dans une mission au-dessus de Berlin. L'enregistrement mettait en avant le sang-froid de l'équipage, leur dévouement et leur courage. $\mathrm{La} \mathrm{BBC}$ mena un sondage auprès des auditeurs qui révéla que ceux-ci avaient été enthousiasmés par le programme ; beaucoup se déclarèrent fortement impressionnés par le courage sans faille des hommes du Bomber Command.

Bien qu'il existât une opposition féroce aux bombardements massifs dans certains cercles intellectuels et religieux, parmi lesquels l'archevêque de Canterbury, Cosmo Lang, ou encore le dramaturge George Bernard Shaw, les voix condamnant l'offensive aérienne stratégique britannique étaient peu nombreuses et peu entendues. Le député travailliste Richard Stokes fut l'un des opposants les plus véhéments à l'offensive aérienne stratégique à la Chambre des Communes. Ses inquiétudes concernaient le sort des populations civiles se retrouvant sous les bombes du fait de la proximité de cibles dites militaires. Interrogé par Richard Stokes en mars 1943, Archibald Sinclair, alors secrétaire d'État à l'Air, déclara :

Les cibles du Bomber Command sont toujours des cibles militaires, mais le bombardement de nuit d'objectifs militaires implique nécessairement le bombardement de la zone dans laquelle ils se trouvent?

Si la question de la moralité des bombardements est régulièrement soulevée au gouvernement, celui-ci ne change pas pour autant de stratégie. Exaspéré, Richard Stokes déclara en mars 1945 à la Chambre des Communes qu'il renonçait à faire abandonner la campagne aérienne stratégique pour des raisons d'ordre moral, et qu'il s'emploierait à en souligner l'inefficacité militaire ${ }^{10}$.

C'est avec le bombardement de Dresde à la fin de la guerre en février 1945 que la question de la moralité - ou de l'immoralité - des bombardements stratégiques prend toute son ampleur. Dresde est devenue un symbole de la violence des bombardements stratégiques sur l'Allemagne, de la même façon que Hiroshima et Nagasaki sont devenues des symboles de la puissance de destruction nucléaire. S’il est un épisode de la Seconde Guerre mondiale, et plus particulièrement de l'offensive aérienne stratégique, qui demeure controversé en Grande-Bretagne, c'est bien celui-ci. Nombreux sont les historiens qui se sont interrogés sur le statut particulier du bombardement de Dresde, qui a eu et a toujours un statut à part dans l'historiographie du Bomber Command et de ses activités. Il n'y a à ce jour toujours pas de réponse définitive ni d'explication à ce qui confère à Dresde ce fort pouvoir évocateur ; le débat demeure ouvert, attisant d'autant plus la curiosité et le questionnement.

9 Débat à la Chambre des Communes, 31 mars 1943, vol. 388, colonne 155. "The targets of Bomber Command are always military, but night-bombing of military objectives necessarily involves bombing the area in which they are situated."

10 Débat à la Chambre des Communes, 6 mars 1945, vol. 408, colonne 1900. Richard Stokes, après avoir décrit l'horreur des bombardements de Dresde, annonce : « Je laisse de côté la question de la moralité. À mon grand désespoir, je dois renoncer à convaincre les gens sur ce sujet-là. » ("I leave out the moral issue. I have given up in despair trying to persuade people on that issue. ") 
Craignant les réactions hostiles des Britanniques, Churchill prit immédiatement ses distances avec le raid et avec la politique de bombardements massifs, qu'il avait pourtant décidée avec son Cabinet de guerre. Dans un document qu'il envoya à l'état-major du ministère de l'Air, il déclara : "La destruction de Dresde remet sérieusement en question la conduite des bombardements alliés ${ }^{11}$. Ce document plongea l'état-major dans la confusion et la consternation, en particulier à la lumière de la demande - l'insistance, même - de Churchill deux mois plus tôt pour que soient bombardées les villes allemandes à l'Est, parmi lesquelles Dresde. Les doutes de Churchill quant à la moralité des bombardements en Allemagne, pour le moins surprenants aux vues de l'enthousiasme que celui-ci à longtemps manifesté pour l'offensive aérienne stratégique, semblent être une tentative de la part du Premier ministre de se désolidariser de cette politique afin de se préserver de toute condamnation morale trop sévère a posteriori. Et en effet, les ouvrages historiques publiés après le raid retiennent que Churchill avait exprimé des doutes à propos de la destruction de Dresde. L'historien Stephen A. Garrett y voit une tentative d'influencer l'histoire afin d'y occuper une place plus glorieuse : Churchill, lui-même historien, semble avoir voulu laisser dans les archives historiques des traces de son inquiétude au sujet des bombardements stratégiques, et plus particulièrement de celui de Dresde. Pour Garrett, les propos du Premier ministre sont un aveu implicite que le Bomber Command était coupable d'actes de guerre immoraux ${ }^{12}$. D'autres interprétations ont été avancées pour expliquer la soudaine crise de conscience de Churchill. En janvier 1945, les Alliés craignaient encore que la guerre ne se prolonge jusqu'en 1946 et que l'effort de guerre allemand soit ravivé. La priorité était donc de mettre l'Allemagne définitivement à genoux en la frappant aussi fort que possible. Toutefois, à la fin du mois de mars, date de la directive controversée de Churchill, l'effondrement de l'Allemagne apparaissait comme inévitable, et il devenait difficilement justifiable de maintenir l'offensive aérienne. Churchill était désormais inquiet des intentions de Staline, et craignait que celui-ci ne respecte pas les accords signés à Yalta. Peut-être Churchill estimait-il que la Grande-Bretagne et les États-Unis auraient un jour besoin du soutien du peuple allemand contre la puissance soviétique ${ }^{13}$.

Pour l'historien britannique Mark Connelly, la perception du Bomber Command par l'opinion publique a été cristallisée par la soudaine prise de distance du Premier ministre et par le manque d'informations au sujet de la véritable nature des raids aériens et des cibles visées ${ }^{14}$. Privé des honneurs et de

11 Frederick Taylor, Dresden: Tuesday 13 February 1945, Londres, Bloomsbury, 2005, p. 130.

12 Steven Garrett, "Political Leardership and Dirty Hands: Winston Churchill and the City Bombing of Germany ", in Cathal J. Nolan, Ethics and Statecraft. The Moral Dimension of International Affairs, deuxième édition, 2010, p. 72.

13 Paul Addison, Churchill. The Unexpected Hero, Oxford, Oxford University Press, 2005, p. 197.

14 Mark Connelly, "Britain and the Debate over RAF Bomber Command's role in the Second 
la reconnaissance accordés à ses pairs, Arthur Harris se retira, et les missions menées pendant la guerre par le Bomber Command furent plongées dans un oubli délibéré afin d'éviter toute remise en question de la conduite britannique de la guerre. Certains anciens du Bomber Command tentèrent de publier leurs mémoires de guerre, mais ceux-ci passèrent inaperçus à côté des récits de bravoure des pilotes du Fighter Command qui n'étaient pas sujet à controverse.

Plusieurs événements contribuèrent à discréditer le Bomber Command dans les années 1960, période de toutes les remises en question. La publication de l'histoire officielle de l'offensive aérienne stratégique en 1961 réfuta en effet partiellement l'efficacité de l'offensive aérienne stratégique britannique $^{15}$. À ces conclusions en demi-teinte s'ajoutèrent les théories du très controversé historien révisionniste britannique David Irving qui publia en 1963 son ouvrage le plus célèbre, The Destruction of Dresden, dans lequel il remettait en cause la nécessité militaire et la légitimité morale des bombardements alliés de la capitale saxonne, soulignant le nombre important de victimes parmi les populations civiles ${ }^{16}$. D'autre part, des événements sur la scène politique nationale et internationale réveillèrent les fantômes du passé ; ainsi, en Grande-Bretagne, le mouvement prônant le désarmement nucléaire, le CND (Campaign for Nuclear Disarmament), créé en 1957 et mené par les personnalités qui avaient combattu les bombardements stratégiques pendant la Seconde Guerre mondiale, centra à nouveau le débat sur le sort des populations civiles en cas de guerre. L'utilisation militaire de l'énergie nucléaire vint à être associée avec le souvenir des bombardements pendant le conflit ${ }^{17}$.

Un deuxième événement, sur la scène internationale cette fois, contribua à influencer le débat sur le recours aux bombardements stratégiques pendant la Seconde Guerre mondiale : la guerre du Vietnam. Bien que la Grande-Bretagne ne participât pas à ce conflit, il suscita un vif débat auprès des Britanniques. L'épisode le plus marquant fut certainement la campagne américaine de bombardements au nord du Vietnam de 1965 à 1968, dont les victimes civiles rappelaient tristement celles des raids aériens britanniques. Le gouvernement d'Harold Wilson, qui devint Premier ministre en 1964, soutenait politiquement le conflit américain et ses objectifs, ce qui incluait le bombardement du nord du Vietnam ; la presse britannique, en revanche, était très critique à l'égard de cette position. De nombreuses manifestations furent organisées en Grande-

World War ", Historische Literatur, 2004, vol. 2, p. 8-12.

15 Charles Webster et Noble Frankland, The Strategic Air Offensive against Germany, t. 3, Londres, HM Stationery Office, 1961, p. 284.

16 David Irving, The Destruction of Dresden, Londres, William Kimber, 1963.

17 Holger Nehring, "Cold War, Apocalypse and Peaceful Atoms. Interpretations of Nuclear Energy in the British and West-German Anti-Nuclear Weapons Movements, 1955-1964 ", Historical Social Review, 2004, vol. 29, p. 150. 
Bretagneen protestation aux bombardements au nord du Vietnam. ${ }^{18}$ Bien qu'au cours des années 1970 et 1980 un certain nombre d'ouvrage historiques et fictionnels tentèrent de mettre en avant les conditions particulièrement éprouvantes des équipages du Bomber Command et les fortes pertes subies par ceux-ci, le débat public entourant les bombardements massifs de l'Allemagne demeurait vivace. En 1984, une statue fut érigée à Londres en l'honneur d'Arthur Harris ; l'inauguration de celle-ci fut troublée par les huées de la foule, et un cordon de police dût être mis en place pendant plusieurs mois afin de protéger la statue des tentatives d'actes de vandalisme dont elle faisait régulièrement l'objet. Les Britanniques semblaient alors encore mal à l'aise avec le souvenir des bombardements stratégiques en Allemagne.

Les ouvrages publiés dans les années 1960 et 1970, dont la plupart insistait sur les souffrances des populations civiles allemandes, présentèrent aux Britanniques la campagne de bombardements sous un jour nouveau, et ceux-ci y furent particulièrement sensibles, probablement en raison du fait que les souvenirs du Blitz, que certains avaient vécu, étaient encore proches. Le jumelage en 1959 des villes de Coventry et Dresde fut un élément de réconciliation important entre la Grande-Bretagne et l'Allemagne, et les Britanniques commencèrent à voir les Allemands comme des victimes d'un régime que tous n'avaient pas soutenu. Le Daily Telegraph écrivait à l'occasion du $25^{\mathrm{e}}$ anniversaire du bombardement de Dresde en février 1970 :

[L]e bien et le mal sont des concepts qui coexistent en Angleterre aussi bien qu'en Allemagne [...]. Les bombardements aériens, et particulièrement l'oblitération de Dresde, nous a conduits à défendre l'indéfendable ${ }^{19}$.

Si les ouvrages publiés dans les années 1970 et au début des années 1980 présentent les membres d'équipage du Bomber Command de façon plus humaine et moins stigmatisée, il n'en va pas de même pour Harris, sur qui les critiques se déchaînent. L'un des exemples les plus marquants est peutêtre l'ouvrage Dresden 1945: The Devil's Tinderbox d'Alexander McKee, publié en 1982, dans lequel l'auteur s'emploie à démontrer que, si la décision de bombarder Dresde avait été prise par l'ensemble des hauts commandants alliés, elle avait été mise en œuvre avec un enthousiasme diabolique par Harris. Il y eut un regain d'intérêt dans les années 1980 pour le Bomber Command, et ce notamment en raison de la mort de Harris le 5 avril 1984, qui engendra inévitablement des débats houleux en Grande-Bretagne.

18 Jonathan Colman, A "special relationship"? Harold Wilson, Lyndon B. Johnson and AngloAmerican Relations "at the summit", 1964-1968, Manchester, Manchester University Press, 2004, p. 122.

19 Alan Russell, "Why Dresden Matters ", in Paul Addison et Jeremy A. Crang, Firestorm. The Bombing of Dresden, 1945, Londres, Pimlico, 2006, p. 165-166. « [R]ight and wrong coexist in concepts in England as well as Germany [...]. Aerial bombing and especially the wiping out of Dresden left the rest of us to defend the indefensible. " 
Les récents conflits auxquels a participé l'armée britannique semblent avoir fortement influencé la perception de Bomber Command par l'opinion publique britannique. La guerre des Malouines en 1982, conflit qui opposa le Royaume-Uni à l'Argentine, eut un impact majeur sur la mentalité britannique. La victoire britannique permit au Royaume-Uni d'affirmer sa souveraineté sur ces territoires, mais aussi de retrouver une certaine fierté nationale après avoir subi pendant trente ans le déclin de son influence sur l'échiquier mondial. La guerre des Malouines fut fédératrice d'un point de vue identitaire puisque la défense d'un territoire britannique était en jeu ; elle permit de renouer avec la gloire militaire britannique. La RAF ne joua qu'un rôle limité dans ce conflit en raison du coût très élevé des opérations aériennes, mais aussi parce que la population des Malouines était majoritairement britannique, ce qui excluait tout bombardement ${ }^{20}$.

Il semble que la guerre du Golfe de 1991, décidée en raison de l'invasion irakienne du Koweït, ait été l'élément déclencheur dans le changement progressif de la perception du Bomber Command par l'opinion publique britannique. Pendant la guerre du Golfe, les Britanniques étaient particulièrement inquiets de l'usage qui pourrait être fait des bombardements par la Royal Air Force. Il apparaît cependant que le rejet des bombardements massifs en tant qu'instruments de guerre acceptables et celui de la légitimité des cibles civiles coïncidèrent avec un changement de doctrine au sein des forces aériennes britanniques elles-mêmes ; celles-ci se concentrèrent sur l'utilisation d'une aviation tactique, utilisant un faible nombre d'appareils multi-rôles de haute efficacité destinés à appuyer les forces navales et terrestres ${ }^{21}$.

Pour la majorité des historiens, les forces aériennes furent utilisées avec une plus grande efficacité au cours de la guerre du Golfe en raison de la supériorité qualitative des appareils modernes en termes de précision ${ }^{22}$. La Royal Air Force déploya six escadrons, basés en Arabie Saoudite. Au total, 158 avions et hélicoptères furent envoyés en Irak, ainsi que 5500 soldats. À titre d'exemple, le seul raid de Dresde avait mobilisé 805 avions de bombardement la nuit du 13 au 14 février $1945^{23}$. Lopinion publique britannique était majoritairement favorable à une action armée en Irak : en février 1991, un sondage du quotidien britannique The Guardian révéla que $80 \%$ des personnes interrogées soutenaient les bombardements en Irak ${ }^{24}$. Ce soutien quasi unanime s'explique peut-être par l'utilisation faite par les médias du conflit, et par les images que ceux-ci décidèrent ou non de diffuser. Les Britanniques, de même

20 Martin Middlebrook, Task Force. The Falklands War, 1982, Londres, Penguin, 1984, p. 120.

21 Markus Mäder, In Pursuit of Conceptual Excellence. The Evolution of British Military-Strategic Doctrine in the Post-Cold War Era, 1989-2002, Berne, Peter Lang, p. 104.

22 Alastair Finlan, The Gulf War of 1991, New York, Rosen, 2009, p. 28.

23 Max Hastings, Bomber Command, Londres, Penguin, édition de 1999, p. 337.

24 Milan Rai, War Plan Iraq. Ten Reasons against War on Iraq, Londres, Verso, 2002, p. 165. 
que les populations des pays membres de l'OTAN participant aux opérations en Irak, étaient en règle générale isolés de la réalité immédiate et des menaces directes de violence. Pendant la Seconde Guerre mondiale, ce n'était qu'après avoir découvert les images des villes allemandes en ruine dans la presse ou dans les "newsreels" au cinéma que les Britanniques avaient pu se rendre compte de l'ampleur de l'offensive aérienne, et s'en étaient émus. Cette réaction peut sembler surprenante de leur part, ayant eux-mêmes vécu les attaques destructrices de la Luftwaffe pendant le Blitz en 1941 puis les attaques des V2 en 1944. Tom Harrison, fondateur de l'organisme d'étude sociale Mass Observation, avait réalisé en 1941 une série de sondages qui mirent en évidence le peu de soutien à l'offensive aérienne stratégique de la part des Britanniques ayant été touchés par les bombardements allemands ${ }^{25}$.

Bien que la guerre du Golfe fût la première à être retransmise en direct sur les télévisions, et de ce fait hautement médiatisée, la population en GrandeBretagne - ainsi que dans les sociétés occidentales - n'avait qu'une vision partielle des événements en Irak. À l'exception de la BBC, qui avait diffusé des images de la destruction du bunker de Saddam Hussein, les médias britanniques ne diffusèrent aucune image et ne publièrent aucune photographie des victimes des attaques des forces de la coalition, préférant montrer des dommages d'ordre matériel. Les historiens Martin Shaw et Roy Carr-Hill vont jusqu’à affirmer que les médias britanniques nièrent de façon délibérée la violence infligée aux Irakiens ${ }^{26}$. En outre, fort peu d'images furent diffusées des soldats de la coalition sur le terrain.

Les médias ont sans nul doute joué un rôle important dans la représentation de la guerre du Golfe, mais il semble que les victimes dans les rangs britanniques aient particulièrement touché l'opinion publique. Au cours du conflit, six appareils de la Royal Air Force furent abattus ; comparé aux autres appareils alliés, ce chiffre est relativement élevé ; à titre d'exemple, les armées de l'air française, italienne et koweitienne ne perdirent qu'un seul appareil - les États-Unis, en revanche, perdirent 29 appareil $s^{27}$. En outre, 47 soldats furent tués au combat. Les Britanniques furent donc confrontés plus d'une fois aux images télévisées des corps rapatriés et aux cérémonies émouvantes qui s'ensuivirent. Ces cérémonies furent d'autant plus nombreuses après l'intervention en Afghanistan en 2001 qui a fait, au moment de la rédaction de cet article, 445 victimes parmi les soldats de l'armée britannique ${ }^{28}$. C'est peut-être cela qui a le plus contribué à

25 Tom Harrison, Living Through the Blitz, Londres, Penguin, édition de 1990, p. 314-316.

26 Martin Shaw et Roy Carr-Hill, " Mass Media Attitudes to the Gulf War in Britain ", Electronic Journal of Communication, $\mathrm{n}^{\circ}$ 2, 1991, sans pagination : http://www.cios.org/ EJCPUBLIC/002/1/00212.HTML, page consultée le 10/04/2012.

27 Matthew Hurley, "Saddam Hussein and Iraqi Air Power: Just Having an Air Force Isn'tEnough ", Airpower Journal, n 4, 1992, p. 14.

28 Rob Crilly, "British Soldier Killed in Afghanistan », le 15 octobre 2013, The Daily Telegraph. 
changer la façon dont le Bomber Command était perçu par l'opinion publique. En effet, comment critiquer les actions des pilotes pendant la Seconde Guerre mondiale alors que des soldats britanniques tombent au combat presque quotidiennement et sont automatiquement célébrés en héros dans les médias ? Une telle différence de traitement ne semblait plus pouvoir tenir dès lors que l'armée britannique soutenait des pertes relativement élevées.

Nous retrouvons le même type de discours avec la seconde guerre du Golfe en 2003, lorsque les Britanniques se joignirent aux Américains pour envahir l'Irak. Le rôle joué par les forces britanniques fut considérablement plus grand que celui joué en 1991. Bien que ce second conflit ne bénéficiât pas du même soutien de l'opinion publique et qu'il fut vivement contesté, les victimes militaires britanniques émurent tout autant les Britanniques. Par ailleurs, les guerres du Golfe faisaient appel à des notions de "britannicité " («Britishness ») et étaient présentées, tant par les politiques que par les médias, comme s'inscrivant dans une longue tradition de nation en guerre, mise en évidence par Linda Colley dans son ouvrage Britons: Forging the Nations 1707-1837, dans lequel celle-ci analyse la façon dont la Grande-Bretagne est devenue un État solidaire en combattant ses ennemis européens ${ }^{29}$. Les médias britanniques firent tout particulièrement référence à la Seconde Guerre mondiale et à ses mythes fédérateurs afin d'attirer la sympathie et l'approbation de l'opinion publique. Étant donné que la Grande-Bretagne est une nation qui continue d'envoyer des hommes au combat, la guerre continue de jouer un rôle important dans la construction identitaire du pays. Bien que les décisions des hommes politiques soient souvent contestées et que l'armée britannique soit une armée professionnelle, les forces armées bénéficient d'une très bonne réputation et du soutien de l'opinion publique.

Ce changement dans la perception de la guerre est certainement à l'origine du regard favorable que les Britanniques portent aujourd'hui au Bomber Command. Les ouvrages publiés depuis la fin des années 2000 n'hésitent pas à employer le terme de " héros " pour décrire les membres du Bomber Command, et insistent fortement sur la grande difficulté de leurs missions ainsi que sur les pertes très élevées qu'ils ont subies. Le peu d'opposition rencontré par le projet de construction du mémorial de Londres atteste d'une évolution sensible dans le débat lié aux bombardements massifs de l'Allemagne pendant la Seconde Guerre mondiale. Celui-ci n'est pas entièrement clos, loin s'en faut : certaines voix se sont élevées contre l'édification du mémorial, que ce soit de façon directe en invoquant le rôle controversé du Bomber Command pendant la guerre, ou de façon plus indirecte en critiquant son architecture et son emplacement. Celui-ci est en effet bien plus imposant que n'importe quel autre lieu de commémoration de la Seconde Guerre mondiale en Grande-Bretagne, et sa situation géographique au cœur de la capitale lui confere une plus grande visibilité. 
La classe politique britannique apporta son soutien unanime au projet, lancé en 2008 par le quotidien The Daily Telegraph. Les courriers des lecteurs des grands quotidiens britanniques furent envahis de lettres de soutien au mémorial, et plusieurs personnalités se mobilisèrent, offrant au Bomber Command toujours plus de visibilité dans les médias. Dans un tel contexte, il aurait certainement été fort délicat pour la classe politique de ne pas afficher son soutien, ce qui aurait fait ainsi également courir le risque de raviver la controverse des bombardements stratégiques au cours de la Seconde Guerre mondiale. Gordon Brown, alors Premier ministre, déclara :

J'ai toujours pensé que les 55000 hommes courageux du Bomber Command, qui sont tombés pour leur pays, méritaient une totale reconnaissance pour leur courage et leur sacrifice ${ }^{30}$.

La famille royale se mobilisa également, ce qui apporta au projet de construction du mémorial une visibilité médiatique supplémentaire. Le 30 octobre 2010, le Prince William en personne signait une lettre publiée dans le Daily Express :

Parmi les 125000 hommes du Bomber Command, 56000 sacrifièrent leur vie pour leur pays et pour la liberté. [...] Le rôle joué par le Bomber Command pendant la Seconde Guerre mondiale fut tellement controversé qu'on en oublie parfois ce que ces jeunes gens ont enduré pour nous. [...] Le mémorial en l'honneur du Bomber Command a mis bien trop de temps à voir le jour ${ }^{31}$.

Les plans du mémorial furent confiés à l'architecte britannique Liam O'Connor, auteur de plusieurs autres mémoriaux de la Seconde Guerre mondiale. L'architecture du mémorial est néo-classique, composée d'une partie centrale, surélevée, entourée de deux ailes, plus basses, formées de sept colonnes indépendantes préfigurant la sculpture des sept aviateurs au centre du mémorial. Celle-ci fut sculptée par Philip Jackson et représente les sept membres d'un équipage, anonyme, du Bomber Command: au centre se trouve le pilote, entouré du mitrailleur dorsal, du navigateur et du mécanicien à sa gauche, ainsi que du mitrailleur arrière, de l'opérateur radio et du bombardier à sa droite. Cinq d'entre eux scrutent l'horizon à la recherche d'un appareil qui ne reviendra vraisemblablement pas de mission, tandis que deux d'entre eux abaissent la tête, en signe de résignation, de tristesse et de

30 The Daily Telegraph, le 28 octobre 2008. "I have always believed that the 55,000 brave men of Bomber Command who lost their lives in the service of their country deserved the fullest recognition of their courage and sacrifice. "

31 The Daily Express, le 30 octobre 2010. L'article fut également publié sur le site web du Prince William : www.princeofwales.gov.uk, page consultée le 20/06/2012. "Of the 125,000 men of Bomber Command, 56,000 gave their lives for this Country and for freedom. [...] So much of the analysis of Bomber Command's role in the Second World War has been mired in controversy that it is sometimes forgotten what these young men went through for us. [...] A memorial to the crews of Bomber Command has been half a century too long in coming. " 
deuil. Les sept aviateurs se tiennent proches les uns des autres, comme unis par un lien inébranlable : ce sont des frères d'armes. Plusieurs inscriptions sont gravées à l'intérieur de la partie centrale du mémorial. À la droite de la sculpture centrale se trouve l'inscription suivante :

Ce mémorial est dédié aux 55573 aviateurs du Royaume-Uni, du Commonwealth et des nations alliées qui ont servi dans le RAF Bomber Command et ont perdu la vie pendant la Seconde Guerre mondiale ${ }^{32}$.

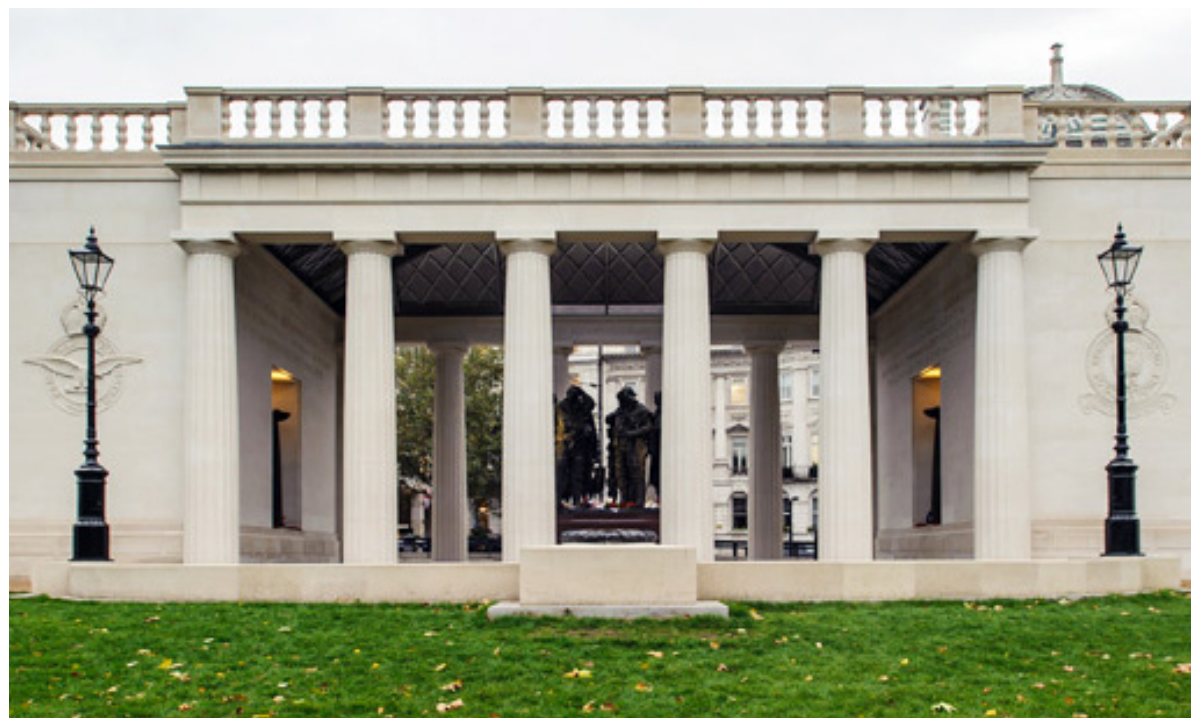

(C) Bomber Command, Memorial Association, 2012. Vue extérieure du mémorial du Bomber Command depuis Green Park.

À la gauche de la sculpture se trouve une citation de Churchill datant de septembre 1940 : "Les chasseurs sont notre salut mais seuls les bombardiers peuvent apporter la victoire ». La dernière inscription, enfin, est une citation de Périclès : «La liberté appartient à ceux qui ont le courage de la défendre "

32 "This memorial is dedicated to the 55,573 airmen from the United Kingdom, British Commonwealth and Allied nations who served in RAF Bomber Command and lost their lives over the course of the Second World War. "

33 "Freedom is the sure possesion of those who have the courage to defend it. " 


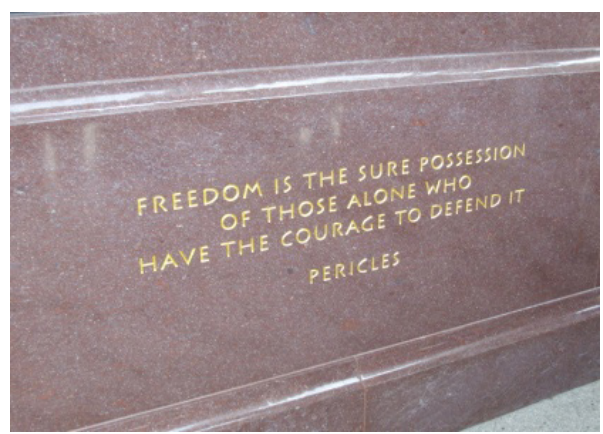

(C) Madeline Zielinski, 2012. Inscription figurant sur le socle de la sculpure centrale du mémorial ("Freedom is the sure possession of those alone who have the courage to defend it. Pericles").
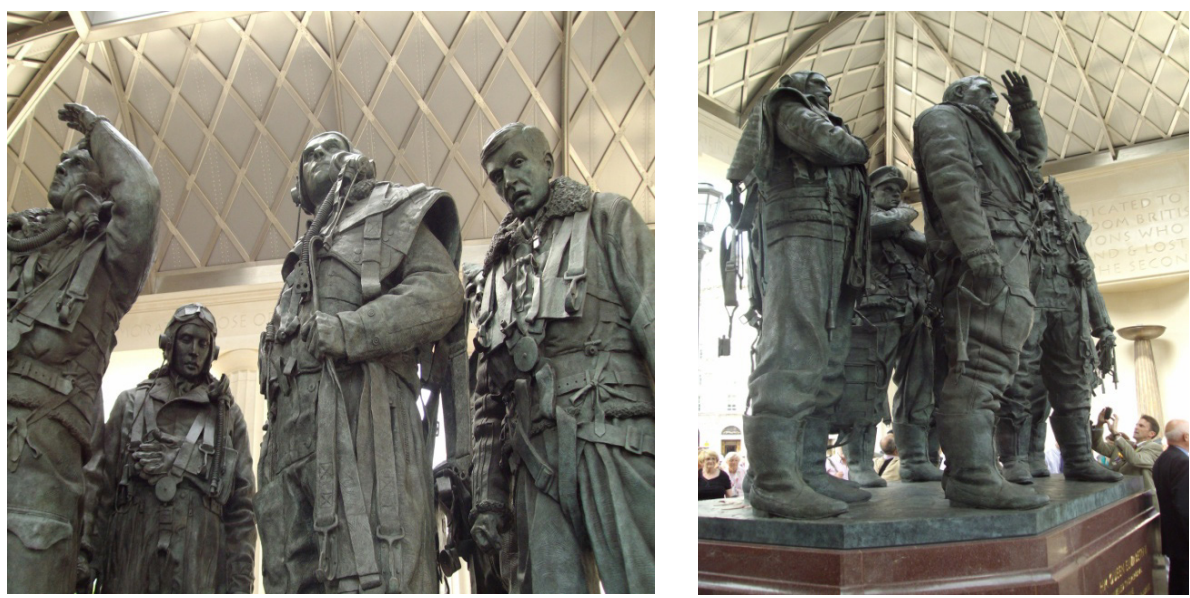

(C) Madeline Zielinski, 2012. Les membres d'équipage, sculptés par Philip Jackson.

Le mémorial du Bomber Command à Green Park est imposant ; sa taille, à elle seule, fait de l'ombre à tous les autres mémoriaux de la Seconde Guerre mondiale de la capitale. Nous pouvons donc nous interroger sur l'importance que l'on a voulu donner à ce monument, de même que sur le message dont celui-ci est porteur. Le critique d'art victorien John Ruskin avait avancé dans ses écrits au $\mathrm{XIX}^{\mathrm{e}}$ siècle la théorie selon laquelle il existe un lien étroit entre l'esthétique de l'architecture d'une société et les valeurs morales de celle-ci ${ }^{34}$. Ainsi l'architecture peut-elle se faire la voix des changements d'une société et des messages que celle-ci veut transmettre. Il semble que le mémorial du Bomber Command soit plus qu'un simple hommage au sacrifice de ses aviateurs : il grave dans la pierre le nouveau statut des équipages, désormais élevés au rang de héros. Après avoir été si longtemps écartés du récit héroïque de la

34 Monroe C. Beardsley, Aesthetics. Problems in the Philosophy of Criticism, Cambridge, Hackett Publishing, 1981, p. 584. 
Seconde Guerre mondiale - "l'heure de gloire » de la Grande-Bretagne, pour reprendre les termes de Churchill - le Bomber Command semble désormais en faire partie intégrante. L'inscription à l'intérieur du mémorial en hommage à toutes les victimes des bombardements, quelle que soit leur nationalité, semble chercher à réconcilier les différentes perspectives sur la campagne de bombardements massifs britanniques : que l'on s'accorde ou non sur leur utilité stratégique, la bravoure des équipages du Bomber Command ne saurait quant à elle être remise en question. Et en effet, cela semble être le discours dominant auprès des historiens britanniques de la Seconde Guerre mondiale. Richard Overy, l'un des principaux détracteurs de l'offensive aérienne stratégique alliée, soulignait quelques jours avant l'inauguration du mémorial sa portée fédératrice :

L'inauguration du mémorial du Bomber Command est peut-être l'occasion de trouver un terrain d'entente dans la question, qui fait toujours polémique, de l'héritage laissé par la Grande-Bretagne en guerre. Il est juste de saluer le sacrifice des quelques 55000 hommes qui périrent, de même que nous honorons le souvenir des morts inutiles des batailles de la Somme ou de Passchendaele ${ }^{35}$.

L'historien et philosophe A.-C. Grayling, qui avait publié en 2006 Among the Dead Cities. Is the Targeting of Civilians in War Ever Justified ?, précise dans l'introduction de la réédition de cet ouvrage en 2014 :

Je salue l'inauguration du mémorial du Bomber Command à Green Park [...] qui rend hommage aux 55000 hommes tués pendant les campagnes de bombardements; l'hommage s'étend à toutes les victimes, dont les jeunes aviateurs font partie ${ }^{36}$.

Tous en revanche ne s'accordent pas sur les choix artistiques des architectes du mémorial, à l'image de l'historien Keith Lowe, qui regrette son extravagance :

Les hommes du Bomber Command méritent [...] un monument à leur image, plus modeste et plus sincère. Ce monolithe triomphaliste en dit plus sur le culte malsain que nous vouons à la guerre que sur ceux qui sont morts pour y mettre $\mathrm{fin}^{37}$.

35 Richard Overy, in The Guardian, le 22 juin 2012. "The opening of the Bomber Command monument is perhaps a moment to try and find some common ground over this unresolved element of Britain's wartime legacy. There is a good case for recognising the sacrifice of the 55000-plus who died, just as we remember the wasted dead at the Somme or Passchendaele. »

36 A.-C. Grayling, Among the Dead Cities. Is the Targeting of Civilians in War Ever Justified?, Londres, Bloomsbury, édition révisée, 2014, p. XI. "When the memorial to Bomber Command was opened in Green Park [...], commemorating the 55,000 men killed in the course of the bombing campaigns, I welcomed it; the tribute is to all the victims, among whom the young air crew members themselves number. »

37 Keith Lowe, "How should we remember the men of Bomber Command? ", le 28 juin 2012. "The men of Bomber Command deserve [...] something more modest, and more honest, like the men themselves. This triumphalist monolith says more about our unhealthy fetishism of the war than it does about the men who died trying to bring it to an early end. » : http://www. historyextra.com/bomber, page consultée le 3/10/2014. 
Si l'utilité stratégique des bombardements massifs est aujourd'hui encore débattue par les historiens en Grande-Bretagne, le mépris dont furent longtemps victimes les équipages du Bomber Command est unanimement condamné, et la bravoure et le sacrifice du Bomber Command ne sauraient être remis en question. Dans les débats historiographiques actuels, les équipages du Bomber Command sont tout autant des victimes que les civils qui périrent sous les bombes alliées. Cette position semble dominer largement les débats publics ; pour la majorité des Britanniques, ce mémorial est la juste récompense des efforts consentis par le Bomber Command et de leur sacrifice -55573 membres périrent, soit $50 \%$ des effectifs. Ces chiffres furent rappelés de nombreuses fois au cours de la cérémonie d'inauguration du mémorial, comme autant de justifications à sa construction. À l'heure où les historiens mettent en avant les mémoires individuelles du conflit, l'offensive aérienne stratégique britannique et la question de la moralité des bombardements massifs apparaissent désormais davantage nuancées, ou tout du moins du point de vue des équipages du Bomber Command qui, après avoir connu la honte et l'oubli, sont aujourd'hui salués en Grande-Bretagne.

Madeline Zielinski

EA 7314 EEE

madeline.zielinski@u-bordeaux.montaigne.fr

\begin{abstract}
Résumé
Longtemps exclus des pratiques commémoratives et de la mémoire de la Seconde Guerre mondiale, les anciens combattants du Bomber Command sont désormais considérés comme des héros en Grande-Bretagne. Cet article examine la façon dont les récents conflits auxquels ont participé les Britanniques a contribué à modifier l'image jusqu'à lors négative des pilotes de bombardiers, et, de façon plus générale, de la représentation de la Seconde Guerre mondiale en Grande-Bretagne.
\end{abstract}

\title{
Mots-clés
}

Mémoire, Seconde Guerre mondiale, bombardements massifs, Bomber Command, Mémorial de Londres.

\begin{abstract}
After being excluded from the national narrative of the Second World War for their role in bombing Germany, the men of Bomber Command are now hailed as heroes in Great-Britain. This essay examines how the conflicts in which Britain has taken part in recent years have changed the way Bomber Command are portrayed and remembered today, and the effect this recent turn of events has had on remembering the Second World War in Britain.
\end{abstract}

Keywords

Memory, Second World War, massive bombing, Bomber Command, London Memorial. 